\title{
TITLE:
}

\section{Charmonium properties in deconfinement phase in anisotropic lattice QCD}

$\operatorname{AUTHOR}(S)$ :

lida, H.; Doi, T.; Ishii, N.; Suganuma, H.; Tsumura, K.

\section{CITATION:}

lida, H. ... [et al]. Charmonium properties in deconfinement phase in anisotropic lattice QCD. Physical Review D 2006, 74(7): 074502.

\section{ISSUE DATE:}

2006-10-30

URL:

http://hdl.handle.net/2433/198862

\section{RIGHT:}

(C) 2006 American Physical Society 
PHYSICAL REVIEW D 74, 074502 (2006)

\title{
Charmonium properties in deconfinement phase in anisotropic lattice QCD
}

\author{
H. Iida, ${ }^{1, *}$ T. Doi ${ }^{2}$ N. Ishii, ${ }^{3}$ H. Suganuma, ${ }^{4}$ and K. Tsumura ${ }^{4}$ \\ ${ }^{1}$ Yukawa Institute for Theoretical Physics, Kyoto University, Kyoto 606-8502, Japan \\ ${ }^{2}$ RIKEN BNL Research Center, Brookhaven National Laboratory, Upton, New York 11973, USA \\ ${ }^{3}$ Department of Physics, The University of Tokyo, Tokyo 113-0033, Japan \\ ${ }^{4}$ Department of Physics, Kyoto University, Kitashirakawaoiwake, Sakyo, Kyoto 606-8502, Japan
}

(Received 5 February 2006; published 30 October 2006)

\begin{abstract}
$J / \Psi$ and $\eta_{c}$ above the QCD critical temperature $T_{c}$ are studied in anisotropic quenched lattice QCD, considering whether the $c \bar{c}$ systems above $T_{c}$ are spatially compact (quasi-)bound states or scattering states. We adopt the standard Wilson gauge action and $O(a)$-improved Wilson quark action with renormalized anisotropy $a_{s} / a_{t}=4.0$ at $\beta=6.10$ on $16^{3} \times(14-26)$ lattices, which correspond to the spatial lattice volume $V \equiv L^{3} \simeq(1.55 \mathrm{fm})^{3}$ and temperatures $T \simeq(1.11-2.07) T_{c}$. We investigate the $c \bar{c}$ system above $T_{c}$ from the temporal correlators with spatially extended operators, where the overlap with the ground state is enhanced. To clarify whether compact charmonia survive in the deconfinement phase, we investigate spatial boundary-condition dependence of the energy of $c \bar{c}$ systems above $T_{c}$. In fact, for low-lying $S$-wave $c \bar{c}$ scattering states, it is expected that there appears a significant energy difference $\Delta E \equiv E(\mathrm{APBC})-E(\mathrm{PBC}) \simeq 2 \sqrt{m_{c}^{2}+3 \pi^{2} / L^{2}}-2 m_{c}$ ( $m_{c}$ : charm quark mass) between periodic and antiperiodic boundary conditions on the finite-volume lattice. In contrast, for compact charmonia, there is no significant energy difference between periodic and antiperiodic boundary conditions. As a lattice QCD result, almost no spatial boundary-condition dependence is observed for the energy of the $c \bar{c}$ system in $J / \Psi$ and $\eta_{c}$ channels for $T \simeq(1.11-2.07) T_{c}$. This fact indicates that $J / \Psi$ and $\eta_{c}$ would survive as spatially compact $c \bar{c}$ (quasi-)bound states below $2 T_{c}$. We also investigate a $P$-wave channel at high temperature with maximal entropy method and find no low-lying peak structure corresponding to $\chi_{c 1}$ at $1.62 T_{c}$.
\end{abstract}

DOI: 10.1103/PhysRevD.74.074502

PACS numbers: $12.38 . \mathrm{Gc}, 12.38 . \mathrm{Mh}, 14.40 . \mathrm{Gx}, 25.75 . \mathrm{Nq}$

\section{INTRODUCTION}

To complete the phase diagram of quantum chromodynamics (QCD) is one of the most challenging attempts in particle physics. The difficulty of QCD originates from the nonperturbative nature in the low-energy region, where the running coupling constant becomes large. As a consequence, color confinement and chiral symmetry breaking occur as nonperturbative phenomena, and the vacuum becomes the hadronic phase. On the other hand, at high temperature or high density region, color deconfinement and chiral symmetry restoration are expected to be realized. This phase is called the quark-gluon-plasma (QGP) phase. Actually, lattice QCD simulations show color deconfinement [1] and chiral symmetry restoration [2] above the QCD critical temperature $T_{c}$. The QGP phase transition is also investigated in various effective models [3-8].

As an important signal of QGP creation, $J / \Psi$ suppression [9-13] was theoretically proposed in the middle of the 80 's $[4,5]$. The basic assumption of $J / \Psi$ suppression is that $J / \Psi$ disappears above $T_{c}$ due to vanishing of the confinement potential and appearance of the Debye screening effect, which are actually shown in lattice QCD simulations $[14,15]$.

Experimentally, QGP search is performed at CERN-SPS and Relativistic Heavy Ion Collider (RHIC) with ultrarela-

*Electronic address: iida@yukawa.kyoto-u.ac.jp tivistic heavy-ion collisions [9-13]. The experiment of NA-50 collaboration at CERN-SPS first reported the anomalous $J / \Psi$ suppression in $\mathrm{Pb}-\mathrm{Pb}$ collision (158 GeV/c per nucleon) [13]. Recently, the RHIC experiments show the various signals of QGP, e.g., $J / \Psi$ suppression [4,5], enhancement of strange particles [16], jet quenching and high $p_{T}$ suppression [17,18], elliptic flow $v_{2}$ [17] and so on, in the collisions of Au-Au $(200 \mathrm{GeV} / c$ per nucleon).

At first, QGP was naïvely speculated as simple quarkgluon gas. Nowadays, there are several indications that QGP is not simple perturbative quark-gluon gas in quenched lattice QCD. For example, it is pointed out that the spatial correlation in $\pi$ and $\sigma$ channels remains even in QGP phase in lattice QCD [19]. The other example is the relation between energy density and pressure, which does not satisfy the Stephan-Boltzmann relation even above $T_{c}$ [14]. The calculation of transport coefficients at finite temperature on quenched lattices also shows the strongly correlated gluon plasma [20]. These simulations indicate that some of the nonperturbative properties may survive in QGP phase. Experimentally, in the reports of RHIC [912], QGP seems to behave as perfect liquid, which strongly interacts, rather than dilute gas, from the comparison of the experiments with numerical simulations of hydrodynamics [21]. The strongly correlated deconfined phase is called strongly coupled QGP phase, and is investigated with much attention. 
Very recently, some lattice QCD calculations indicate an interesting possibility that $J / \Psi$ and $\eta_{c}$ seem to survive even above $T_{c}$ [22-25]. In Ref. [22], the authors calculate correlators of charmonia at finite temperature and find the strong spatial correlation between $c$ and $\bar{c}$ even above $T_{c}$. In Refs. [23-25], the authors extract spectral functions of charmonia from temporal correlators at high temperature using the maximal entropy method (MEM). Although there are some quantitative differences, the peaks corresponding to $J / \Psi$ and $\eta_{c}$ seem to survive even above $T_{c}\left(T_{c}<T<\right.$ $2 T_{c}$ ) in the $c \bar{c}$ spectral function.

However, all of these calculations may suffer from a possible problem that the observed $c \bar{c}$ state on lattices is not a nontrivial charmonium but a trivial $c \bar{c}$ scattering state, because it is difficult to distinguish these two states in lattice QCD. One of the reasons of the difficulty is that a narrow peak does not immediately indicate a spatially compact (quasi-)bound state. In QGP phase, the potential between $q$ and $\bar{q}$ is considered to be the Yukawa potential due to the Debye screening [14]. Therefore, the binding energy of $q$ and $\bar{q}$ above $T_{c}$ may be small. Then, the bound state of $q$ and $\bar{q}$ may not be spatially compact. In addition, MEM has a relatively large error, which sometimes leads to uncertainty for the structure of the spectral function.

In this paper and our previous proceeding [26], using lattice QCD, we aim to clarify whether the $c \bar{c}$ system above $T_{c}$ is a spatially compact (quasi-)bound state or a scattering state, which is spatially spread. To distinguish these two states, we investigate spatial boundary-condition dependence of the energy of the $c \bar{c}$ system by comparing results on periodic and antiperiodic boundary conditions. If the $c \bar{c}$ system is a scattering state, there appears an energy difference $\Delta E$ between the two boundary conditions as $\Delta E \simeq 2 \sqrt{m_{c}^{2}+3 \pi^{2} / L^{2}}-2 m_{c}$ with the charm quark mass $m_{c}$ on a finite-volume lattice with $L^{3}$ (see Sec. II). If the $c \bar{c}$ system is a spatially compact (quasi-)bound state, the boundary-condition dependence is expected to be small even in finite volume. In Ref. [27], by changing the spatial periodicity of (anti-)quarks, the authors actually try to distinguish between a scattering state and a spatially compact resonance.

In this study, we use anisotropic lattice QCD with anisotropy $a_{s} / a_{t}=4.0$. The reason why we use the anisotropic lattice is as follows: At finite temperature $T$, the temporal lattice size is restricted to $0 \leq t \leq 1 / T$. Then, in calculating the temporal correlator $G(t)$ at high temperature, we cannot take the sufficient number of points for $G(t)$. By using anisotropic lattice, more data points are available for $G(t)$. For the accurate measurement of $G(t)$, we use such a technical improvement of lattice QCD.

For further technical improvement, we use spatially extended operators with hadron size in the actual lattice calculations at high temperature. We are interested in the low-lying spectrum in the $c \bar{c}$ systems at high temperature in this study, since the ground-state component is desired to dominate in the range of $0 \leq t \leq 1 / T$. For this purpose, we use the spatially extended operator to enhance the ground-state overlap.

This paper is organized as follows: In Sec. II, we discuss the method to distinguish a spatially compact (quasi)bound state from a scattering state by changing the spatial boundary condition for (anti-)quarks. In Sec. III, we briefly explain anisotropic lattice QCD. In Sec. IV, we show the method to extract the energy of the ground state of $c \bar{c}$ systems from temporal correlators at finite temperature in lattice QCD. Section V shows lattice QCD results of $J / \Psi$ and $\eta_{c}$ above $T_{c}$. Using the method discussed in Sec. II, we find the survival of $J / \Psi$ and $\eta_{c}$ as spatially compact (quasi-)bound states above $T_{c}\left(\sim 2 T_{c}\right)$. In Sec. VI, we perform the MEM analysis for the $c \bar{c}$ systems in $J / \Psi, \eta_{c}$, and $\chi_{c 1}$ channels above $T_{c}$ using the lattice QCD data. Section VII is devoted to conclusion and outlook.

\section{METHOD TO DISTINGUISH COMPACT STATES FROM SCATTERING STATES}

In this section, we explain the method to distinguish compact states from scattering states in terms of their spatial extension. In Sec. II A, we discuss and estimate the energy shift of a state due to the change of spatial boundary condition for the $c \bar{c}$ systems. In Sec. II B, we discuss the correction from a short-range potential. As a result, we find that the correction is small compared with the energy shift in the $c \bar{c}$ scattering state above $T_{c}$.

\section{A. Boundary-condition dependence and energy shift for $c \bar{c}$ scattering states}

For the distinction between compact states and scattering states, we investigate the $c \bar{c}$ system on the periodic boundary condition $(\mathrm{PBC})$ and on the antiperiodic boundary condition (APBC), respectively, and examine spatial boundary-condition dependence for the $c \bar{c}$ system. Here, in the PBC and the APBC cases, we impose periodic and antiperiodic boundary condition for (anti-)quarks on a finite-volume lattice, respectively.

For a compact $c \bar{c}$ (quasi-)bound state, the wave function of the $c \bar{c}$ system is spatially localized and insensitive to spatial boundary conditions in lattice QCD as shown in Fig. 1(a), so that the charmonium behaves as a compact boson and its energy on APBC is almost the same as that on PBC [27].

For a $c \bar{c}$ scattering state, the wave function is spatially spread and sensitive to spatial boundary conditions as shown in Fig. 1(b), and hence there emerges the energy difference between $\mathrm{PBC}$ and $\mathrm{APBC}$ due to the nonzero relative momentum of $c$ and $\bar{c}$ on APBC even in the lowest energy state. On PBC, the momentum of a quark or an antiquark is discretized as

$$
p_{k}=\frac{2 n_{k} \pi}{L}\left(k=1,2,3, n_{k} \in \mathbf{Z}\right)
$$


PBC

(a)

Bound State
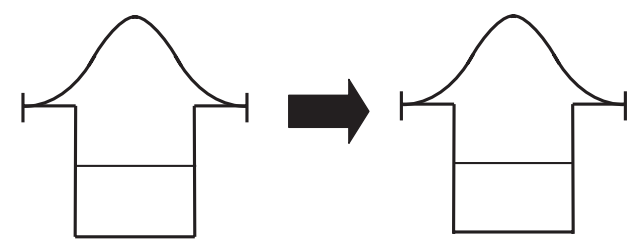

(b)

Scattering State
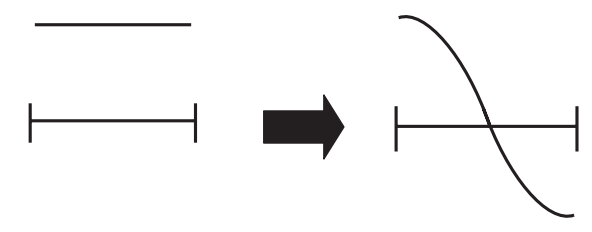

FIG. 1. Schematic figures for boundary-condition dependence of $c \bar{c}$ wave functions: (a) the bound-state case and (b) the scattering-state case in terms of PBC and APBC. (a) For the bound state, the wave function is spatially localized, and it is insensitive to the spatial boundary condition. Hence, the basic properties of the spatially localized bound state are almost the same between PBC and APBC. (b) For the scattering state, the wave function is spatially spread, and therefore it is sensitive to the spatial boundary condition in a finite-size box. In particular, a drastic change occurs for the wave function of the low-lying scattering state between PBC and APBC cases.

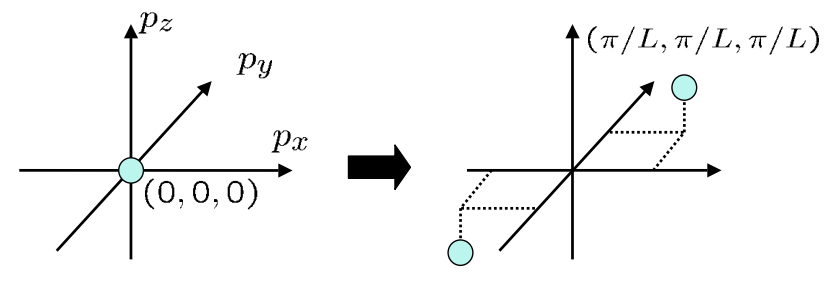

$(-\pi / L,-\pi / L,-\pi / L)$

PBC for $\mathrm{C} \overline{\mathrm{C}}$ scattering state $\quad$ APBC for $\mathrm{c} \overline{\mathrm{C}}$ scattering state

FIG. 2 (color online). Pictorial expression of minimum momentum of the (anti-)quark on PBC and APBC in the case of $c \bar{c}$ scattering state in the center of mass flame. $c$ and $\bar{c}$ are in the finite-size box with the spatial volume $L^{3}$. On PBC, both particles have zero lowest momentum in the lowest state. On the other hand, on APBC, quark and antiquark have an opposite nonzero momentum. on the finite lattice with the spatial volume $L^{3}$. Therefore, on PBC, the minimum momentum is $\vec{p}_{\text {min }}=\overrightarrow{0}$. On APBC, the (anti-)quark momentum is discretized as

$$
p_{k}=\frac{\left(2 n_{k}+1\right) \pi}{L}\left(k=1,2,3, n_{k} \in \mathbf{Z}\right) .
$$

In this case, the (anti-)quark momentum cannot take zero even in the lowest energy state. Then, the minimum (anti)quark momentum is

$$
\left|\vec{p}_{\min }\right|=\frac{\sqrt{3} \pi}{L}
$$

as is depicted in Fig. 2. Thus, there is an energy difference between PBC and APBC for the lowest $c \bar{c}$ scattering state. Neglecting the interaction between $c$ and $\bar{c}$, the energy difference is estimated as

$$
\Delta E \simeq 2 \sqrt{m_{c}^{2}+\vec{p}_{\min }^{2}}-2 m_{c}=2 \sqrt{m_{c}^{2}+3 \pi^{2} / L^{2}}-2 m_{c}
$$

where $m_{c}$ is charm quark mass. The minimum momentum of a quark and an antiquark in the case of a scattering state is depicted in Fig. 2.

Table I summarizes the mass of the $c \bar{c}$ compact bound state (charmonia) and the energy of the $c \bar{c}$ scattering state both on PBC and APBC. In the case of the $c \bar{c}$ scattering state, there emerges the energy difference between $\mathrm{PBC}$ and APBC.

Such a method, to distinguish a compact (quasi-)bound state from a scattering state by changing boundary conditions, is actually used in Ref. [27]. This method is essentially based on the finiteness of lattice volume. Note that the finite volume is used in several studies for the analyses of a scattering state and/or a compact bound state in lattice QCD [28-30].

\section{B. Correction from a short-range potential}

Here, we discuss the possible correction to the energy difference of a scattering state between PBC and APBC from a short-range potential. In the previous subsection, we neglect the interaction between $c$ and $\bar{c}$ and estimate the energy difference between $\mathrm{PBC}$ and $\mathrm{APBC}$ for the $c \bar{c}$ scattering state. However, in the actual situation above

TABLE I. Summary of the boundary-condition dependence of the compact charmonia and the $c \bar{c}$ scattering state. The energy difference $\Delta E$ is calculated on the spatial lattice size $L=$ $1.55 \mathrm{fm}$ and the charm quark mass $m_{c} \simeq 1.3 \mathrm{GeV}$.

\begin{tabular}{lcc}
\hline \hline & Charmonia & $c \bar{c}$ scattering state \\
\hline Mass (energy) on PBC & Bound-state mass $M$ & $\simeq 2 m_{c}$ \\
Mass (energy) on APBC & Bound-state mass $M$ & $\simeq 2 \sqrt{m_{c}^{2}+\vec{p}_{\text {min }}^{2}}$ \\
$\Delta E \equiv E($ APBC) $-E(\mathrm{PBC})$ & $\simeq 0$ & $\simeq 2 \sqrt{m_{c}^{2}+\vec{p}_{\text {min }}^{2}}-2 m_{c} \simeq 0.35 \mathrm{GeV}$ \\
\hline \hline
\end{tabular}


$T_{c}$, a quark and antiquark interact with each other by the Yukawa potential

$$
V(r)=-A \frac{e^{-m(T) r}}{r},
$$

where $m(T)$ is the temperature-dependent Debye screening mass [14,31]. The prefactor $A$ corresponds to the Coulomb coefficient at $T=0$ and is estimated as $A \simeq 0.28$ [32]. Therefore, the simple estimation of the energy shift $\Delta E$ obtained in Sec. II A may be corrected in the presence of the Yukawa potential. In the following, considering the Yukawa potential $V(r)$, we estimate the energy shift $\Delta E$ of the $c \bar{c}$ scattering state in the nonrelativistic quantum mechanics.

Consider two particles, $c$ and $\bar{c}$, in the finite box with $x$, $y, z \in[0, L]$ where the boundary condition is periodic or antiperiodic. $c$ and $\bar{c}$ interact each other with the Yukawa potential in Eq. (5). We estimate the lowest energy of the $c \bar{c}$ scattering state in $\mathrm{PBC}$ and $\mathrm{APBC}$ cases, respectively.

We use the variational method for the charmonium wave function $\psi(\vec{r})(\vec{r}$ : the relative coordinate) in this estimate. We prepare appropriate basis $\phi_{i}(\vec{r})$ and expand the wave function $\psi(\vec{r})$ as

$$
\psi(\vec{r})=\sum_{i} C_{i} \phi_{i}(\vec{r})
$$

where the coefficients $\left\{C_{i}\right\}$ satisfy $\sum_{i}\left|C_{i}\right|^{2}=1$, for the orthonormal basis $\phi_{i}(\vec{r})$. The Hamiltonian in the $c \bar{c}$ system is given by

$$
\hat{H}=-\frac{1}{2 \mu}\left(\frac{\partial^{2}}{\partial x^{2}}+\frac{\partial^{2}}{\partial y^{2}}+\frac{\partial^{2}}{\partial z^{2}}\right)+V(r),
$$

where $\mu=m_{c} / 2$ is the reduced mass of the $c \bar{c}$ system. The energy of the system is expressed by

$$
E \equiv \frac{\int_{V} d^{3} r \psi^{\dagger}(\vec{r}) \hat{H} \psi(\vec{r})}{\int_{V} d^{3} r \psi^{\dagger}(\vec{r}) \psi(\vec{r})} .
$$

Differentiating Eq. (8) by $C_{i}$ and imposing the stationary condition for $E$, i.e., $\frac{\partial E}{\partial C_{i}}=0$, we get the equation for $E$ and $C_{i}$ as

$$
\sum_{j} C_{j}\left\{\int_{V} d^{3} r \phi_{i}^{\dagger}(\vec{r}) \hat{H} \phi_{j}(\vec{r})-E \int_{V} d^{3} r \phi_{i}^{\dagger}(\vec{r}) \phi_{j}(\vec{r})\right\}=0 .
$$

From the condition that $\left\{C_{i}\right\}$ have nontrivial solutions, $E$ is determined as

$$
\operatorname{det}\left(H_{i j}-E S_{i j}\right)=0,
$$

where $\quad H_{i j} \equiv \int_{V} d^{3} r \phi_{i}^{\dagger}(\vec{r}) \hat{H} \phi_{j}(\vec{r}) \quad$ and $\quad S_{i j} \equiv$ $\int_{V} d^{3} r \phi_{i}^{\dagger}(\vec{r}) \phi_{j}(\vec{r})$. Solving Eq. (10), the energy of the $c \bar{c}$ scattering state is obtained.
We take the $i$ th basis as follows:

$$
\begin{aligned}
\phi_{i}(\vec{r})= & \sqrt{\frac{\left(2-\delta_{n_{x} 0}\right)\left(2-\delta_{n_{y} 0}\right)\left(2-\delta_{n_{z} 0}\right)}{L^{3}}} \cos \left(\frac{2 \pi n_{x}}{L} x\right) \\
& \times \cos \left(\frac{2 \pi n_{y}}{L} y\right) \cos \left(\frac{2 \pi n_{z}}{L} z\right),
\end{aligned}
$$

where the normalization factor is set so as to satisfy the orthonormal condition, $\int d^{3} r \phi_{i}^{\dagger} \phi_{j}=\delta_{i j}$. In the PBC case, we choose the basis as

$$
\begin{aligned}
\phi_{0}: \vec{n} & =(0,0,0), \\
\phi_{1}, \phi_{2}, \phi_{3}: \vec{n} & =(1,0,0),(0,1,0),(0,0,1), \\
\phi_{4}, \phi_{5}, \phi_{6}: \vec{n} & =(0,1,1),(1,0,1),(1,1,0) \\
& \cdots
\end{aligned}
$$

Note that $\phi_{1}, \phi_{2}$, and $\phi_{3}$ degenerate and so on. In the $\mathrm{APBC}$ case, we choose the basis as

$$
\begin{gathered}
\phi_{0}: \vec{n}=\left(\frac{1}{2}, \frac{1}{2}, \frac{1}{2}\right), \\
\phi_{1}, \phi_{2}, \phi_{3}: \vec{n}=\left(\frac{3}{2}, \frac{1}{2}, \frac{1}{2}\right),\left(\frac{1}{2}, \frac{3}{2}, \frac{1}{2}\right),\left(\frac{1}{2}, \frac{1}{2}, \frac{3}{2}\right), \\
\phi_{4}, \phi_{5}, \phi_{6}: \vec{n}=\left(\frac{1}{2}, \frac{3}{2}, \frac{3}{2}\right),\left(\frac{3}{2}, \frac{1}{2}, \frac{3}{2}\right),\left(\frac{3}{2}, \frac{3}{2}, \frac{1}{2}\right) \\
\cdots .
\end{gathered}
$$

In the following, we consider the $S$-wave case. Because of the spherical symmetry of $S$ wave, the wave function $\psi$ is invariant under the replacement of $x \leftrightarrow y, y \leftrightarrow z, z \leftrightarrow x$. Therefore, for instance, the coefficients of $\phi_{1}, \phi_{2}$, and $\phi_{3}$ coincide, and one finds $C_{1}=C_{2}=C_{3}, C_{4}=C_{5}=C_{6}$ and so on, both in PBC and APBC cases. The convergence for the number of basis, $N_{\text {basis }}$, is checked and the difference of the energy of lowest state is found to be less than $1 \mathrm{MeV}$ between $N_{\text {basis }}=7$ and 13 .

We use $A=0.28$ as the Coulomb coefficient in the $T=$ 0 case. We adopt $m(T)=g T$ as the result of the lowestorder perturbative QCD calculation at finite temperature [33]. For the QCD coupling constant $g$, we use $g=1$ as a typical value in the infrared region, which corresponds to the scale in the typical lattice QCD simulations with $\beta \equiv$ $\frac{2 N_{c}}{g^{2}} \sim 6$. In this calculation, we consider the case at $T=$ $300 \mathrm{MeV}$, which is slightly above $T_{c}$ in quenched QCD. The mass of charm quark is set by $m_{c}=1.5 \mathrm{GeV}$.

Table II is the summary of the results. $E_{0}^{\text {free }}$ denotes the energy of the lowest state in free case. $E_{0}^{\mathrm{Y}}$ denotes the lowest-state energy in the Yukawa potential. Note that the energy difference $\left|E_{0}^{\mathrm{Y}}-E_{0}^{\mathrm{free}}\right|$ is rather small as $7.8 \mathrm{MeV}$ in the PBC case and $13.7 \mathrm{MeV}$ in APBC case, i.e., about $10 \mathrm{MeV}$. (Even in the extreme case of the Coulomb poten- 
TABLE II. The lowest energy of the $c \bar{c}$ scattering state above $T_{c}$ on PBC and APBC. $E_{0}^{\text {free }}$ and $E_{0}^{\mathrm{Y}}$ denote the lowest energy in the no interaction (free) and the Yukawa potential cases, respectively (the origin of the energy is shifted by $2 m_{c}$ in this nonrelativistic estimation). The energy difference from the free case, $E_{0}^{\mathrm{Y}}-E_{0}^{\text {free }}$, is also shown.

\begin{tabular}{lccc}
\hline \hline & $E_{0}^{\text {free }}$ & $E_{0}^{\mathrm{Y}}$ & $E_{0}^{\mathrm{Y}}-E_{0}^{\text {free }}$ \\
\hline PBC & $0 \mathrm{MeV}$ & $-7.8 \mathrm{MeV}$ & $-7.8 \mathrm{MeV}$ \\
APBC & $318.4 \mathrm{MeV}$ & $304.7 \mathrm{MeV}$ & $-13.7 \mathrm{MeV}$ \\
\hline \hline
\end{tabular}

tial, i.e., $m(T)=0$, where the potential effect is clearly overestimated, the correction is found to be $50 \mathrm{MeV}$ at most.)

Thus, the correction from the short-range potential about $10 \mathrm{MeV}$ is small enough compared with the energy shift $\Delta E \simeq 350 \mathrm{MeV}$ between $\mathrm{PBC}$ and $\mathrm{APBC}$ shown in Table I. Therefore, as far as we consider the $c \bar{c}$ scattering state above $T_{c}$, short-range interaction between $c$ and $\bar{c}$ can be neglected.

Here, we note that the parameter set used in this calculation gives the upper limit of the correction from the shortrange Yukawa interaction. As the temperature increases, the Debye screening mass $m(T)$ becomes larger and the "Coulomb coefficient" $A(T)$ becomes slightly smaller. These tendencies make the interaction smaller at high temperature. Thus, using $A(T)$ at $T=0$ and setting the lowest temperature $T \simeq T_{c}$, which realize the QGP phase, we get the upper limit of the correction from the shortrange interaction. In addition, the use of the perturbative estimation $m(T) \simeq g T$ also leads an "overestimation" for the effect of the Yukawa potential. For, the lattice QCD calculation [31] shows that the actual Debye screening is estimated as $m(T)=(2-3) g T$ near $T_{c}$, and the $q \bar{q}$ potential seems to be more screened in comparison with the perturbative result. In fact, the obtained correction about $10 \mathrm{MeV}$ is reduced in the realistic case. Through the above considerations, the correction obtained in the calculation can be regarded as the upper limit and the actual value of the correction should be smaller than the limit about $10 \mathrm{MeV}$.

\section{ANISOTROPIC LATTICE QCD}

In this paper, we adopt anisotropic lattice QCD for the study of high-temperature QCD, as was mentioned in Sec. I. We explain anisotropic lattice QCD in the following.
For the gauge field, we adopt the standard plaquette action on an anisotropic lattice as [27,34]

$$
\begin{aligned}
S_{G}= & \frac{\beta}{N_{c}} \frac{1}{\gamma_{G}} \sum_{s, i<j \leq 3} \operatorname{Re} \operatorname{Tr}\left\{1-P_{i j}(s)\right\} \\
& +\frac{\beta}{N_{c}} \gamma_{G} \sum_{s, i \leq 3} \operatorname{Re} \operatorname{Tr}\left\{1-P_{i 4}(s)\right\},
\end{aligned}
$$

where $P_{\mu \nu}$ denotes the plaquette operator. In the simulation, we take $\beta \equiv 2 N_{c} / g^{2}=6.10$ and the bare anisotropy $\gamma_{G}=3.2103$, which lead to the renormalized anisotropy as $a_{s} / a_{t}=4.0$. The scale is set by the Sommer scale as $r_{0}^{-1}=395 \mathrm{MeV}$. Then, the spatial and the temporal lattice spacing are evaluated as $a_{s}^{-1} \simeq 2.03 \mathrm{GeV}$ (i.e., $a_{s} \simeq$ $0.097 \mathrm{fm}$ ), and $a_{t}^{-1} \simeq 8.12 \mathrm{GeV}$ (i.e., $a_{t} \simeq 0.024 \mathrm{fm}$ ), respectively. The adopted lattice sizes are $16^{3} \times(14-26)$, which correspond to the spatial lattice size as $L \simeq$ $1.55 \mathrm{fm}$ and the temperature as $(1.11-2.07) T_{c}$. Here, the critical temperature is estimated as $T_{c}=(260-280) \mathrm{MeV}$ at the quenched level [35]. We use 999 gauge configurations, which are picked up every 500 sweeps after the thermalization of 20000 sweeps. We adopt the jackknife error estimate for lattice data.

For quarks, we use $O(a)$-improved Wilson (clover) action on the anisotropic lattice as [27,34]

$$
\begin{aligned}
S_{F} \equiv & \sum_{x, y} \bar{\psi}(x) K(x, y) \psi(y), \\
K(x, y) \equiv & \delta_{x, y}-\kappa_{t}\left\{\left(1-\gamma_{4}\right) U_{4}(x) \delta_{x+\hat{4}, y}\right. \\
& \left.+\left(1+\gamma_{4}\right) U_{4}^{\dagger}(x-\hat{4}) \delta_{x-\hat{4}, y}\right\} \\
& -\kappa_{s} \sum_{i}\left\{\left(r-\gamma_{i}\right) U_{i}(x) \delta_{x+\hat{i}, y}\right. \\
& \left.+\left(r+\gamma_{i}\right) U_{i}^{\dagger}(x-\hat{i}) \delta_{x-\hat{i}, y}\right\}-\kappa_{s} c_{E} \sum_{i} \sigma_{i 4} F_{i 4} \delta_{x, y} \\
& -r \kappa_{s} c_{B} \sum_{i<j} \sigma_{i j} F_{i j} \delta_{x, y},
\end{aligned}
$$

which is an anisotropic version of the Fermilab action [36]. $\kappa_{s}$ and $\kappa_{t}$ denote the spatial and the temporal hopping parameters, respectively, and $r$ the Wilson parameter. $c_{E}$ and $c_{B}$ are the clover coefficients. The tadpole improvement is done by the replacement of $U_{i}(x) \rightarrow U_{i}(x) / u_{s}$, $U_{4}(x) \rightarrow U_{4}(x) / u_{t}$, where $u_{s}$ and $u_{t}$ are the mean-field values of the spatial and the temporal link variables, respectively. The parameters $\kappa_{s}, \kappa_{t}, r, c_{E}, c_{B}$ are to be tuned so as to keep the Lorentz symmetry up to $O\left(a^{2}\right)$. At the

TABLE III. The lattice parameters and related quantities in our anisotropic lattice QCD calculation with $O(a)$-improved Wilson quarks for the effective-mass analysis.

\begin{tabular}{lcccccccc}
\hline \hline$\beta$ & Lattice size & $a_{s}^{-1}$ & $a_{t}^{-1}$ & $\gamma_{G}$ & $u_{s}$ & $u_{t}$ & $\gamma_{F}$ & $\kappa$ \\
\hline 6.10 & $16^{3} \times(14-26)$ & $2.03 \mathrm{GeV}$ & $8.12 \mathrm{GeV}$ & 3.2103 & 0.8059 & 0.9901 & 4.0 & 0.112 \\
\hline \hline
\end{tabular}


tadpole-improved tree level, this requirement leads to $r=$ $a_{t} / a_{s}, c_{E}=1 /\left(u_{s} u_{t}^{2}\right), c_{B}=1 / u_{s}^{3}$ and the tuned fermionic anisotropy $\gamma_{F} \equiv\left(u_{t} \kappa_{t}\right) /\left(u_{s} \kappa_{s}\right)=a_{s} / a_{t}$. For the charm quark, we take $\kappa=0.112$ with $1 / \kappa \equiv 1 /\left(u_{s} \kappa_{s}\right)-2\left(\gamma_{F}+\right.$ $3 r-4)$, which corresponds to the hopping parameter in the isotropic lattice. The bare quark mass $m_{0}$ in spatial lattice unit is expressed as $m_{0}=\frac{1}{2}\left(\frac{1}{\kappa}-8\right)$. We summarize the lattice parameters and related quantities in Table III for the effective-mass analysis. With the present lattice QCD setup, the masses of $J / \Psi, \eta_{c}$, and $\chi_{c 1}$ are calculated as $m_{J / \Psi} \simeq 3.07 \mathrm{GeV}, m_{\eta_{c}} \simeq 2.99 \mathrm{GeV}$, and $m_{\chi_{c 1}} \simeq$ $3.57 \mathrm{GeV}$ at $T \simeq 0$. These values are almost the same as the experimental ones, $m_{J / \Psi}(\exp )=3.10 \mathrm{GeV}, m_{\eta_{c}}(\exp )=$ $2.98 \mathrm{GeV}$, and $m_{\chi_{c 1}}(\exp )=3.51 \mathrm{GeV}$.

\section{TEMPORAL CORRELATORS OF $c \bar{c}$ SYSTEMS AT FINITE TEMPERATURE ON ANISOTROPIC LATTICE}

To investigate the low-lying state at high temperature from the temporal correlator, it is practically desired to use a "good" operator with a large overlap with ground state (ground-state overlap), due to limitation of the temporal lattice size. To this end, we use a spatially extended operator of the Gaussian type as

$$
O(\vec{x}, t) \equiv N \sum_{\vec{y}} \exp \left\{-\frac{|\vec{y}|^{2}}{2 \rho^{2}}\right\} \bar{c}(\vec{x}+\vec{y}, t) \Gamma c(\vec{x}, t)
$$

with the extension radius $\rho$ as the hadronic size in the Coulomb gauge [27,34]. $N$ is a normalization constant. $\Gamma=\gamma_{k}(k=1,2,3)$ and $\Gamma=\gamma_{5}$ correspond to $1^{-}(J / \Psi)$ and $0^{-}\left(\eta_{c}\right)$ channels, respectively. Note here that this form (14) is suitable for $S$-wave states [see Fig. 3(a)]. In the actual calculation, the size parameter $\rho$ is optimally chosen in terms of the ground-state overlap. The energy of the lowlying state is calculated from the temporal correlator,
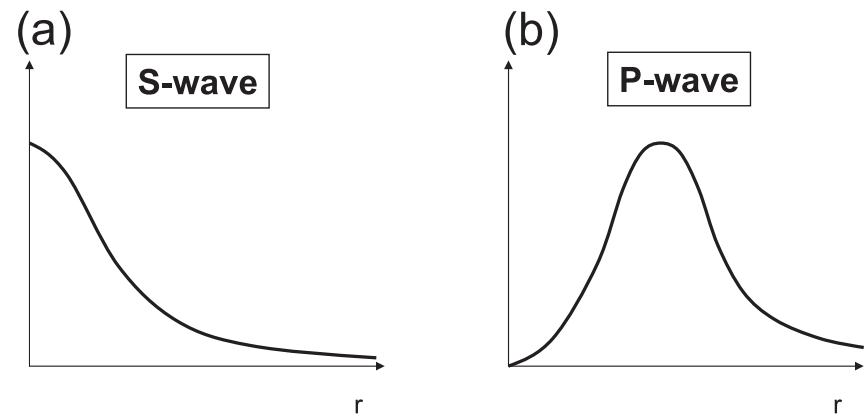

FIG. 3. Schematic figures for the radial wave functions of $c \bar{c}$ compact bound states for (a) the $S$-wave state and (b) the $P$-wave state. The $S$-wave state can be approximated with a Gaussian form $e^{-r^{2} / 2 \rho^{2}}$. In contrast, the wave function of $P$-wave state should be zero at $r=0$ and spatially spreads due to the centrifugal potential.

$$
G(t) \equiv \frac{1}{V} \sum_{\vec{x}}\left\langle O(\vec{x}, t) O^{\dagger}(\overrightarrow{0}, 0)\right\rangle,
$$

where the total spatial momentum of the $c \bar{c}$ system is projected to be zero.

In accordance with the temporal periodicity at finite temperature, we define the effective-mass $m_{\mathrm{eff}}(t)$ from the correlator $G(t)$ by the cosh-type function as [35]

$$
\frac{G(t)}{G(t+1)}=\frac{\cosh \left[m_{\mathrm{eff}}(t)\left(t-N_{t} / 2\right)\right]}{\cosh \left[m_{\mathrm{eff}}(t)\left(t+1-N_{t} / 2\right)\right]},
$$

with the temporal lattice size $N_{t}$. If the correlator $G(t)$ is saturated by the lowest level, it behaves as

$$
G(t) \simeq A \cosh \left[m_{0}\left(t-\frac{N_{t}}{2}\right)\right]
$$

where $m_{0}$ is the energy of the lowest state. In this case, the solution $m_{\text {eff }}(t)$ in Eq. (16) coincides with the lowest-state energy $m_{0}$ in Eq. (17).

To find the optimal value of the extension radius $\rho$, we set two criteria. First, an effective-mass $m_{\text {eff }}(t)$ of a temporal correlator $G(t)$ with $\rho$ should have plateau region in terms of $t$. Second, the ground-state component should be large enough in the plateau region of $m_{\mathrm{eff}}(t)$. For the estimation of the ground-state overlap, we define $g_{\text {fit }}(t)$ as [35]

$$
g_{\text {fit }}(t) \equiv A^{\prime} \cosh \left[m^{\prime}\left(t-N_{t} / 2\right)\right],
$$

where $A^{\prime}$ and $m^{\prime}$ are determined by fitting $g_{\text {fit }}(t)$ to $G(t) / G(0)$ in the plateau region. In general, $g_{\text {fit }}(0) \leq 1$ is satisfied, and $g_{\text {fit }}(0)=1$ is achieved if and only if $G(t)$ is completely dominated by the ground-state contribution in the whole region of $0 \leq t \leq 1 / T$. Therefore, we use $g_{\text {fit }}(0)$ as an index of the ground-state overlap. Namely, if $g_{\text {fit }}(0)$ with $\rho_{1}$ is larger than that with $\rho_{2}$, we regard the operator with $\rho_{1}$ as a better operator which has larger ground-state overlap than that with $\rho_{2}$. Under these criteria, we determine the optimal operator in the range of $\rho=0(0.1 \mathrm{fm})$ $0.5 \mathrm{fm}$. For $\rho=0$ and $0.1 \mathrm{fm}$, the effective masses are found to have no plateau region. Accordingly, we abandon these extension radii. Next, we examine $g_{\text {fit }}(0)$ for $\rho=$ $(0.2-0.5) \mathrm{fm}$, and find that the optimal size of $\rho$ is $\rho=$ $(0.2-0.3) \mathrm{fm}$ at all temperatures for $c \bar{c}$ systems. Actually, the results of $\rho=0.2 \mathrm{fm}$ and $0.3 \mathrm{fm}$ are almost the same. Hereafter, we show the numerical results for $\rho=0.2 \mathrm{fm}$.

\section{LATTICE QCD RESULTS FOR $\boldsymbol{J} / \Psi$ AND $\eta_{c}$ CHANNELS ABOVE $T_{c}$}

We investigate the $c \bar{c}$ systems above $T_{c}$ both in $J / \Psi\left(J^{P}=1^{-}\right)$and $\eta_{c}\left(J^{P}=0^{-}\right)$channels in lattice QCD. For each channel, we calculate the temporal correlator $G(t)$ following Eq. (15), where the total spatial momentum is projected to be zero. From $G(t)$, we extract the effective-mass $m_{\mathrm{eff}}(t)$ both on PBC and APBC, and examine their spatial boundary-condition dependence. From the 
CHARMONIUM PROPERTIES IN DECONFINEMENT ...
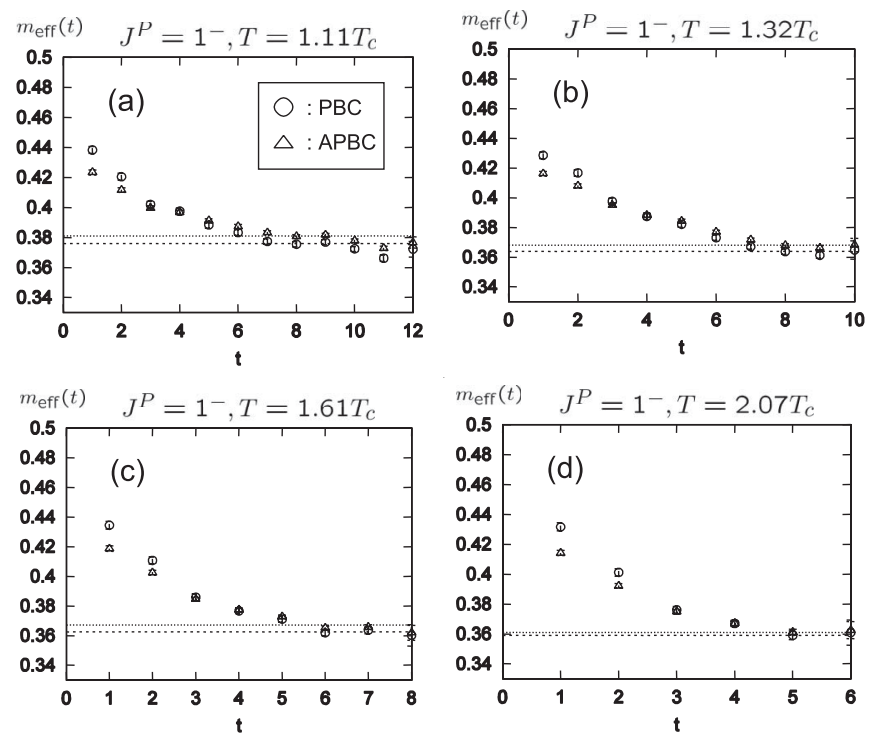

FIG. 4. The effective-mass $m_{\text {eff }}$ is plotted against $t$ for $J / \Psi$ at (a) $1.11 T_{c}$, (b) $1.32 T_{c}$, (c) $1.61 T_{c}$, and (d) $2.07 T_{c}$ in the lattice unit with $a_{t}=(8.12 \mathrm{GeV})^{-1}$. The circles and the triangles denote the results on PBC and APBC, respectively. The dashed and dotted lines denote $E(\mathrm{PBC})$ and $E(\mathrm{APBC})$ obtained from the best-fit analysis, respectively.

cosh-type fit for the correlator $G(t)$ in the plateau region of effective-mass $m_{\text {eff }}(t)$, we extract the energies, $E(\mathrm{PBC})$ and $E(\mathrm{APBC})$, of the low-lying $c \bar{c}$ system on $\mathrm{PBC}$ and $\mathrm{APBC}$, respectively. In fact, if the $c \bar{c}$ system is a spatially compact bound state, one can expect $E(\mathrm{PBC}) \simeq E(\mathrm{APBC})$, which coincides with the bound state mass. On the other hand, if the $c \bar{c}$ system is a scattering state, $E(\mathrm{PBC})$ and $E(\mathrm{APBC})$ are expected to be the corresponding thresholds of the $c \bar{c}$ scattering state, i.e., $E(\mathrm{PBC}) \simeq 2 m_{c}$ and $E(\mathrm{APBC}) \simeq$ $2 \sqrt{m_{c}^{2}+\vec{p}_{\min }^{2}}$.

Figure 4 shows effective-mass plots of $J / \Psi$ on $\mathrm{PBC}$ and APBC for (1.11-2.07) $T_{c}$ in lattice QCD. Table IV shows the boundary-condition dependence of the energy of the $c \bar{c}$ system in the $J / \Psi$ channel for $(1.11-2.07) T_{c}$. It is remarkable that almost no spatial boundary-condition dependence is found for the low-lying energy of the $c \bar{c}$ system, i.e., $\Delta E \equiv E(\mathrm{APBC})-E(\mathrm{PBC}) \simeq 0$. This contrasts to the $c \bar{c}$
PHYSICAL REVIEW D 74, 074502 (2006)

$$
M_{J / \Psi}(T)[\mathrm{GeV}]
$$

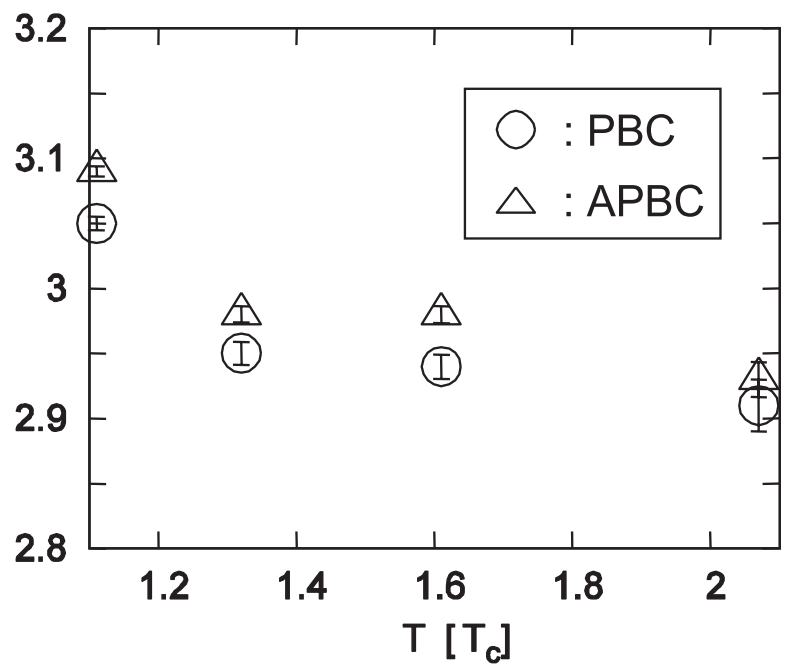

FIG. 5. Temperature dependence of the pole-mass (energy) $M_{J / \Psi}(T)$ of $J / \Psi$ for $(1.11-2.07) T_{c}$ on PBC (circles) and APBC (triangles). The circles and the triangles correspond to $E(\mathrm{PBC})$ and $E(\mathrm{APBC})$ of the $c \bar{c}$ system, respectively. The energy difference $\Delta E \equiv E(\mathrm{APBC})-E(\mathrm{PBC}) \simeq(0.02-0.04) \mathrm{GeV}$ between $\mathrm{PBC}$ and $\mathrm{APBC}$ are considerably smaller than that of the $c \bar{c}$ scattering state, $\Delta E \simeq 0.35 \mathrm{GeV}$.

scattering case of $\Delta E \simeq 2 \sqrt{m_{c}^{2}+3 \pi^{2} / L^{2}}-2 m_{c} \simeq$ $0.35 \mathrm{GeV}$ for $L \simeq 1.55 \mathrm{fm}$ and $m_{c} \simeq 1.3 \mathrm{GeV}$ as was discussed in Sec. II. This result indicates that $J / \Psi$ survives for (1.11-2.07) $T_{c}$ as spatially compact (quasi-)bound state. Figure 5 shows the temperature dependence of the pole mass of $J / \Psi, M_{J / \Psi}(T)$.

Figure 6 shows effective-mass plots of $\eta_{c}$ on PBC and APBC for $(1.11-2.07) T_{c}$. Table $\mathrm{V}$ summarizes the $c \bar{c}$ system in the $\eta_{c}$ channel on $\mathrm{PBC}$ and $\mathrm{APBC}$ at each temperature. Again, almost no spatial boundary-condition dependence is found as $\Delta E \equiv E(\mathrm{APBC})-E(\mathrm{PBC}) \simeq 0$. This result indicates that $\eta_{c}$ also survives for (1.11-2.07) $T_{c}$ as a spatially compact (quasi-)bound state. Figure 7 shows the temperature dependence of the pole mass of $\eta_{c}, M_{\eta_{c}}(T)$.

From Figs. 4 and 6, one finds that $m_{\text {eff }}(t)$ on APBC is saturated by the low-lying state more rapidly than that on

TABLE IV. The energy of the $c \bar{c}$ system in the $J / \Psi$ channel $\left(J^{P}=1^{-}\right)$on PBC and APBC at $\beta=6.10$ and $\rho=0.2 \mathrm{fm}$ at each temperature. The statistical errors are smaller than $0.01 \mathrm{GeV}$. We list also uncorrelated $\chi^{2} / N_{\mathrm{DF}}$ and $\Delta E \equiv E(\mathrm{APBC})-E(\mathrm{PBC})$.

\begin{tabular}{lcccc}
\hline \hline Temperature & Fit range & $E(\mathrm{PBC})\left[\chi^{2} / N_{\mathrm{DF}}\right]$ & $E(\mathrm{APBC})\left[\chi^{2} / N_{\mathrm{DF}}\right]$ & $\Delta E$ \\
\hline $1.11 T_{c}$ & $7-11$ & $3.05 \mathrm{GeV}[0.14]$ & $3.09 \mathrm{GeV}[0.61]$ & $0.04 \mathrm{GeV}$ \\
$1.32 T_{c}$ & $8-11$ & $2.95 \mathrm{GeV}[0.34]$ & $2.98 \mathrm{GeV}[0.33]$ & $0.03 \mathrm{GeV}$ \\
$1.61 T_{c}$ & $6-9$ & $2.94 \mathrm{GeV}[0.10]$ & $2.98 \mathrm{GeV}[0.22]$ & $0.04 \mathrm{GeV}$ \\
$2.07 T_{c}$ & $5-7$ & $2.91 \mathrm{GeV}[0.03]$ & $2.93 \mathrm{GeV}[0.04]$ & $0.02 \mathrm{GeV}$ \\
\hline \hline
\end{tabular}



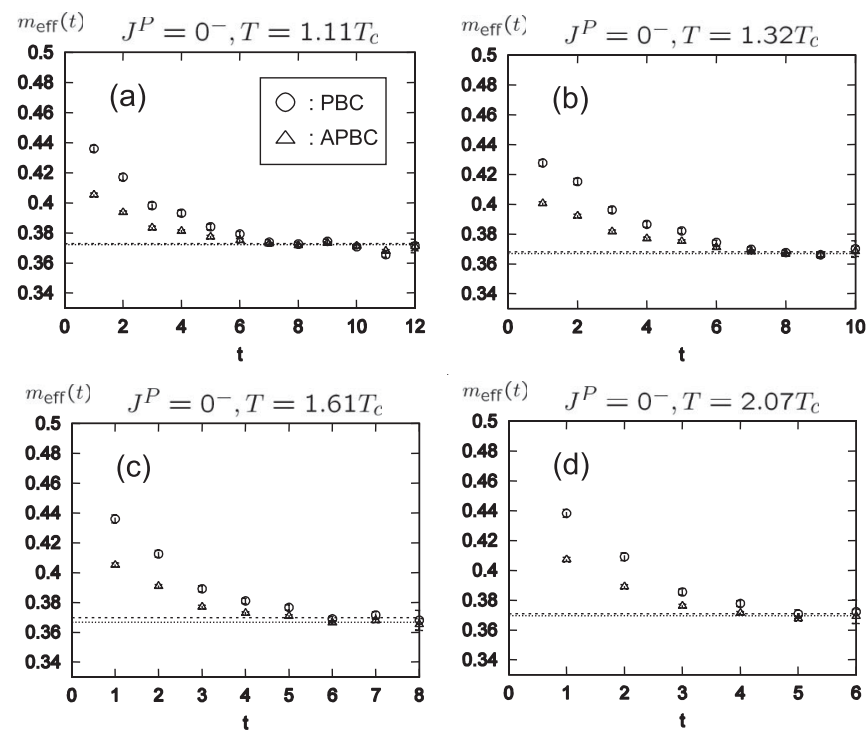

FIG. 6. The effective-mass $m_{\text {eff }}(t)$ is plotted against $t$ for $\eta_{c}$ at (a) $1.11 T_{c}$, (b) $1.32 T_{c}$, (c) $1.61 T_{c}$, and (d) $2.07 T_{c}$ in the lattice unit with $a_{t} \simeq(8.12 \mathrm{GeV})^{-1}$. The circles and the triangles denote the results on $\mathrm{PBC}$ and $\mathrm{APBC}$, respectively. The dashed and the dotted lines denote $E(\mathrm{PBC})$ and $E(\mathrm{APBC})$ obtained from the best-fit analysis, respectively.

PBC, i.e., $m_{\mathrm{eff}}^{\mathrm{PBC}}(t)>m_{\mathrm{eff}}^{\mathrm{APC}}(t)$ for small $t$ and $m_{\mathrm{eff}}^{\mathrm{PBC}}(t) \simeq$ $m_{\mathrm{eff}}^{\mathrm{APC}}(t)$ for large $t$. This fact may be explained as follows: In the channels of $J / \Psi$ and $\eta_{c}$, there are scattering states in addition to the bound state. On APBC, the low-lying scattering states are shifted to higher energy region in contrast to the compact bound state. Thus, the contributions of scattering states dump rapidly on APBC. The (slight) smallness of errorbars on APBC compared with those on PBC may be explained by the same reason (see Figs. 5 and 7).

In Fig. 8, we compare $M_{J / \Psi}(T)$ with $M_{\eta_{c}}(T)$ on PBC. It is interesting that there occurs the inversion of $M_{J / \Psi}(T)$ and $M_{\eta_{c}}(T)$ above $1.3 T_{c}$. In fact, $M_{J / \psi}(T)$ decreases as temperature increases, while $M_{\eta_{c}}(T)$ is almost unchanged (see Fig. 5 and 7). We observe the significant reduction of $M_{J / \Psi}(T)$ of the order of $100 \mathrm{MeV}$. (We note that thermal width broadening also leads to the same effect of the polemass reduction [35].) In any case, it would be interesting to investigate the possible change of the $J / \Psi$ mass above $T_{c}$.

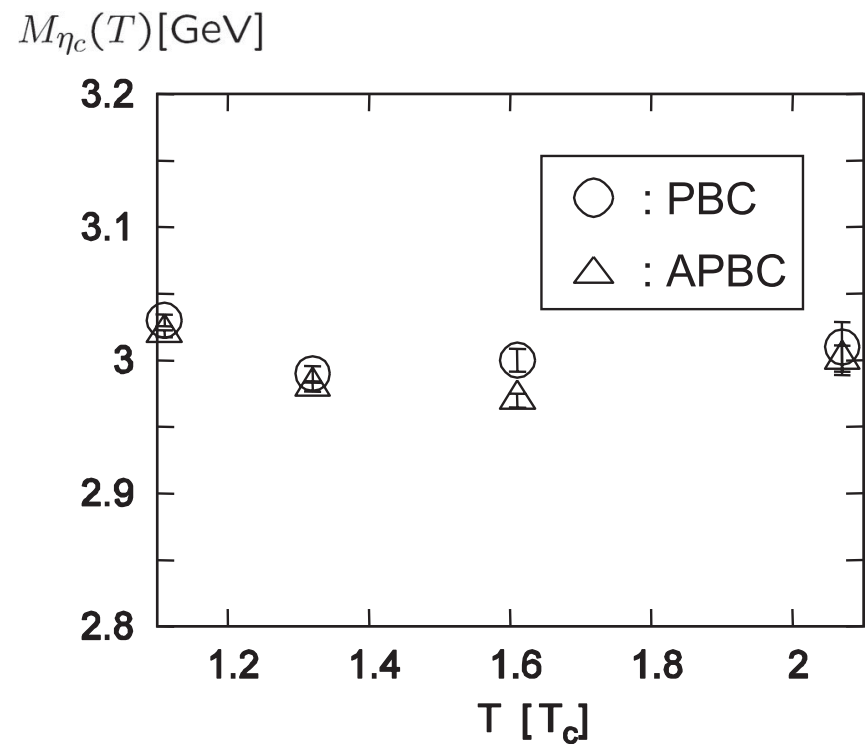

FIG. 7. Temperature dependence of the pole mess (energy) $M_{\eta_{c}}(T)$ of $\eta_{c}$ for $(1.11-2.07) T_{c}$ on PBC (circles) and APBC (triangles). The energy difference $\Delta E \simeq 0$ between $\mathrm{PBC}$ and $\mathrm{APBC}$ are also considerably smaller than that of the $c \bar{c}$ scattering state as well as the $J / \Psi$ case.

\section{MEM ANALYSIS FOR $J / \Psi, \eta_{c}$, AND $\chi_{c 1}$ CHANNELS ABOVE $T_{c}$}

In this section, we perform the MEM analysis for the $c \bar{c}$ systems in $J / \Psi\left(J^{P}=1^{-}\right), \eta_{c}\left(J^{P}=0^{-}\right)$, and $\chi_{c 1}\left(J^{P}=\right.$ $1^{+}$) channels above $T_{c}$ using the lattice QCD data. Here, the axial-vector $\chi_{c 1}$ is a $P$-wave $c \bar{c}$ meson, and its wave function tends to spread due to the centrifugal potential $l(l+1) / m_{c} r^{2}$ ( $r$ : relative distance between $c$ and $\bar{c}, l$ : orbital angular momentum) compared with $S$-wave states such as $J / \Psi$ and $\eta_{c}$ (see Fig. 3). According to this spread wave function, $\chi_{c 1}$ is expected to be sensitive to vanishing of the linear potential and appearance of the Debye screening effect above $T_{c}$. In fact, the dissociation temperature of $\chi_{c 1}$ would be lower than that of $J / \Psi$ and $\eta_{c}$. Therefore, we study the $P$-wave $c \bar{c}$ meson $\chi_{c 1}$ at finite temperature in lattice QCD.

Here, we note that the difficulty with the extended operator in axial-vector channel. Because $\chi_{c 1}$ is a $P$-wave state, the wave function of $\chi_{c 1}$ is not spherical. Moreover, its radial wave function should be zero at the

TABLE V. The energy of the $c \bar{c}$ system in the $\eta_{c}$ channel $\left(J^{P}=0^{-}\right)$on PBC and APBC at $\beta=6.10$ and $\rho=0.2 \mathrm{fm}$ at each temperature. The statistical errors are smaller than $0.01 \mathrm{GeV}$. We list also uncorrelated $\chi^{2} / N_{\mathrm{DF}}$ and $\Delta E \equiv E(\mathrm{APBC})-E(\mathrm{PBC})$.

\begin{tabular}{lcccc}
\hline \hline Temperature & Fit range & $E(\mathrm{PBC})\left[\chi^{2} / N_{\mathrm{DF}}\right]$ & $E(\mathrm{APBC})\left[\chi^{2} / N_{\mathrm{DF}}\right]$ & $\Delta E$ \\
\hline $1.11 T_{c}$ & $7-11$ & $3.03 \mathrm{GeV}[0.04]$ & $3.02 \mathrm{GeV}[0.17]$ & $-0.01 \mathrm{GeV}$ \\
$1.32 T_{c}$ & $7-11$ & $2.99 \mathrm{GeV}[0.78]$ & $2.98 \mathrm{GeV}[0.82]$ & $-0.01 \mathrm{GeV}$ \\
$1.61 T_{c}$ & $6-9$ & $3.00 \mathrm{GeV}[0.31]$ & $2.97 \mathrm{GeV}[0.38]$ & $-0.03 \mathrm{GeV}$ \\
$2.07 T_{c}$ & $5-7$ & $3.01 \mathrm{GeV}[0.03]$ & $3.00 \mathrm{GeV}[0.07]$ & $-0.01 \mathrm{GeV}$ \\
\hline \hline
\end{tabular}




$$
M(T)[\mathrm{GeV}]
$$

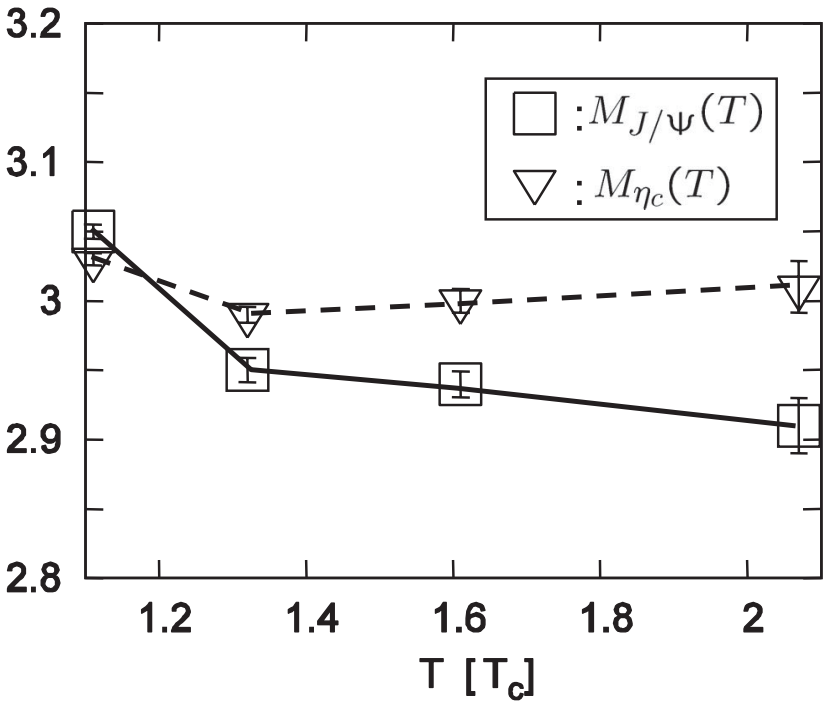

FIG. 8. Temperature dependence of the pole mass (on PBC) of $J / \Psi$ and $\eta_{c}$ for (1.11-2.07) $T_{c}$. The squares denote $M_{J / \Psi}(T)$ and the inverse triangles denote $M_{\eta_{c}}(T)$. There occurs the level inversion of $J / \Psi$ and $\eta_{c}$ above $1.3 T_{c}$.

origin due to the centrifugal potential, as shown in Fig. 3(b). Therefore, the spherical and Gaussian type extension of the operator, which is suitable for an $S$-wave state [see Fig. 3(a)], may not be a good choice to investigate a possible $P$-wave bound state of $\chi_{c 1}$. Actually, we calculate the effective mass with Gaussian extended operator with $\rho=(0-0.5) \mathrm{fm}$ in $\chi_{c 1}$ channel above $T_{c}$ and find that the effective mass has no plateau region. This fact indicates that we cannot extract the low-lying energy state clearly in $\chi_{c 1}$ channel with the operator.

Instead, we perform the analysis of charmonia including $\chi_{c 1}$ above $T_{c}$ with the maximal entropy method [23-25] with the local interpolating field $O_{\Gamma}(\vec{x}, t)=\bar{c} \Gamma c(\Gamma=$ $\left.\gamma_{k}, \gamma_{5}, \gamma_{5} \gamma_{k}\right)$. Using MEM, we can extract the spectral function $A(\omega)$ from the temporal correlator $G(t) \equiv$ $\frac{1}{V} \sum_{\vec{x}}\left\langle O_{\Gamma}(\vec{x}, t) O_{\Gamma}^{\dagger}(\overrightarrow{0}, 0)\right\rangle$. [Here, we define $G(t)$ as the spinaveraged temporal correlator for vector and axial-vector channels.] At finite temperature $T$, the spectral function $A(\omega)$ is related to $G(t)$ as

$$
\begin{aligned}
G(t) & =\int_{0}^{\infty} d \omega K(t, \omega) A(\omega), \\
K(t, \omega) & =\left(e^{-t \omega}+e^{-(1 / T-t) \omega}\right) /\left(1-e^{-\omega / T}\right) .
\end{aligned}
$$

Then, obtaining $A(\omega)$ is carried out by solving the inverse problem. In the standard framework of MEM, one introduces an appropriate "default function" $m(\omega)$ for the spectral function $A(\omega)$ and constructs the Shannon-Janes entropy,

$$
S=\int_{0}^{\infty}\left[A(\omega)-m(\omega)-A(\omega) \log \left(\frac{A(\omega)}{m(\omega)}\right)\right] d \omega,
$$

which plays an essential role in the MEM analysis [37]. For the default function $m(\omega)$, we adopt the spectral function in the lowest perturbative calculation of QCD, i.e., $m(\omega)=$ $m_{0} \omega^{2}$ with $m_{0}=3 / 8 \pi^{2}$ for the pseudoscalar $\left(\eta_{c}\right)$ channel and $m_{0}=1 / 4 \pi^{2}$ (spin-averaged) for the vector $(J / \Psi)$ and the axial-vector $\left(\chi_{c 1}\right)$ channels. Since the asymptotic behavior of QCD can be well described with perturbative QCD, $A(\omega)$ tends to approach $m(\omega)$ in the large $\omega$ region.

For the MEM analysis, we adopt the Wilson quark action in anisotropic lattice QCD. Here, instead of the improvement of the action, we use the finer lattice with lattice spacing $a_{t}=a_{s} / 4=(20.2 \mathrm{GeV})^{-1}=9.75 \times$ $10^{-3} \mathrm{fm}$ with $\beta=7.0$ and $\gamma_{G}=3.5$. The spatial and temporal hopping parameters are chosen to be $\kappa_{s}=$ 0.08285 and $\kappa_{t}=0.28799$, i.e., $\gamma_{F}=\kappa_{t} / \kappa_{s}=3.476$, so as to reproduce low-lying charmonium masses $\left(m_{J / \Psi} \simeq\right.$ $3.10 \mathrm{GeV}, m_{\eta_{c}} \simeq 3.03 \mathrm{GeV}$ ) at $T=0$ [23]. The lattice size is $20^{3} \times 46$, which corresponds to the lattice volume $L^{3} \simeq(0.78 \mathrm{fm})^{3}$ and the temperature $T=1.62 T_{c}$. The lattice parameters and related quantities for the MEM analysis are summarized in Table VI.

To begin with, we perform the MEM analysis for $J / \Psi$ and $\eta_{c}$ above $T_{c}$ using the local interpolating fields, $O_{J / \Psi}=\bar{c} \gamma_{k} c$ and $O_{\eta_{c}}=\bar{c} \gamma_{5} c$, respectively. Figures 9 and 10 show the spectral function of the $J / \Psi$ and $\eta_{c}$ channels at $T=1.62 T_{c}$, respectively. For each channel, the MEM analysis has been done for both PBC and APBC cases. In Figs. 9 and 10, we observe a clear peak around $4 \mathrm{GeV}$, which would correspond to the bound-state pole of $J / \Psi$ and $\eta_{c}$, respectively. Here, we note that the appearance of the peak structure is highly nontrivial, because the default function of MEM is a perturbative one denoted above, which does not have the peak structure. No difference between PBC and APBC is observed for $J / \Psi$ and $\eta_{c}$, which indicates that $J / \Psi$ and $\eta_{c}$ appear as spatially localized compact bound states. These results of $J / \Psi$ and $\eta_{c}$ are consistent with those obtained in previous sections. The peaks in the high-energy region $(\omega>5 \mathrm{GeV})$ are considered as lattice artifacts $[25,38]$.

TABLE VI. The lattice parameters and related quantities in our lattice calculations with Wilson quarks for the MEM analysis.

\begin{tabular}{lccccccc}
\hline \hline$\beta$ & Lattice size & $a_{s}^{-1}$ & $a_{t}^{-1}$ & $\gamma_{G}$ & $\gamma_{F}$ & $\kappa_{s}$ & $\kappa_{t}$ \\
\hline 7.0 & $20^{3} \times 46$ & $5.05 \mathrm{GeV}$ & $20.2 \mathrm{GeV}$ & 3.5 & 3.476 & 0.08285 & 0.28799 \\
\hline \hline
\end{tabular}



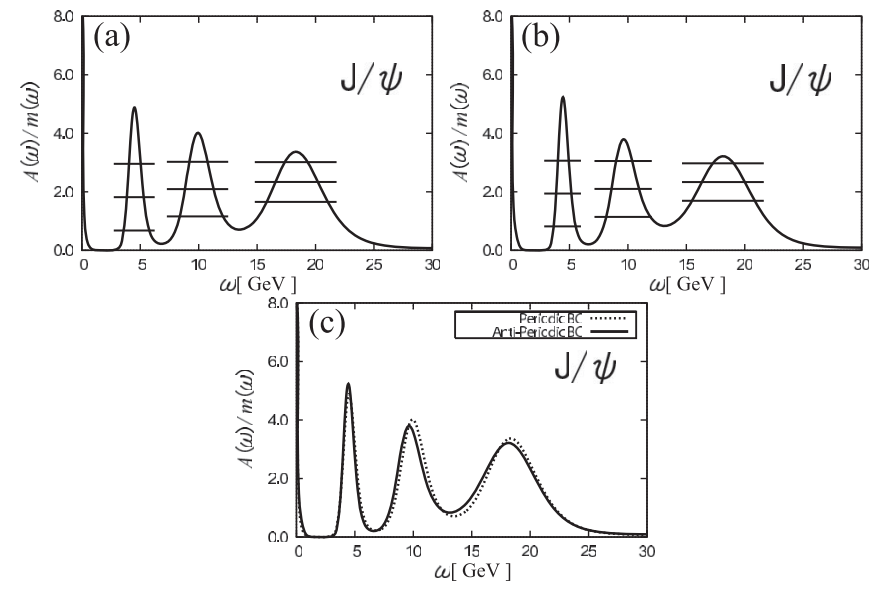

FIG. 9. The spectral function $A(\omega)$ of the $c \bar{c}$ state in vector $\left(J^{P}=1^{-}\right)$channel on (a) PBC and (b) APBC at $1.62 T_{c}$ extracted with MEM from lattice QCD data of the temporal correlator. $m(\omega)$ denotes the default function of MEM [23-25]. The statistical error is denoted by the three solid lines. There is a low-lying peak which corresponds to $J / \Psi\left(m_{J / \Psi} \simeq 3.1 \mathrm{GeV}\right)$ even above $T_{c}$. We add (c) as the comparison between PBC (dotted line) and APBC (solid line). The results almost coincide between PBS and APBC cases.

As a caution, we note that our MEM result for each peak includes the statistical error denoted by three solid lines in Figs. 9 and 10. Because of the uncertainty in $J / \Psi$ and $\eta_{c}$ channels, the lowest peak seems to be broadened and located around $4 \mathrm{GeV}$, which is somehow different from the expected peak position of $J / \Psi$ and $\eta_{c}$, about $3 \mathrm{GeV}$. We speculate that this relatively large error mainly origi-

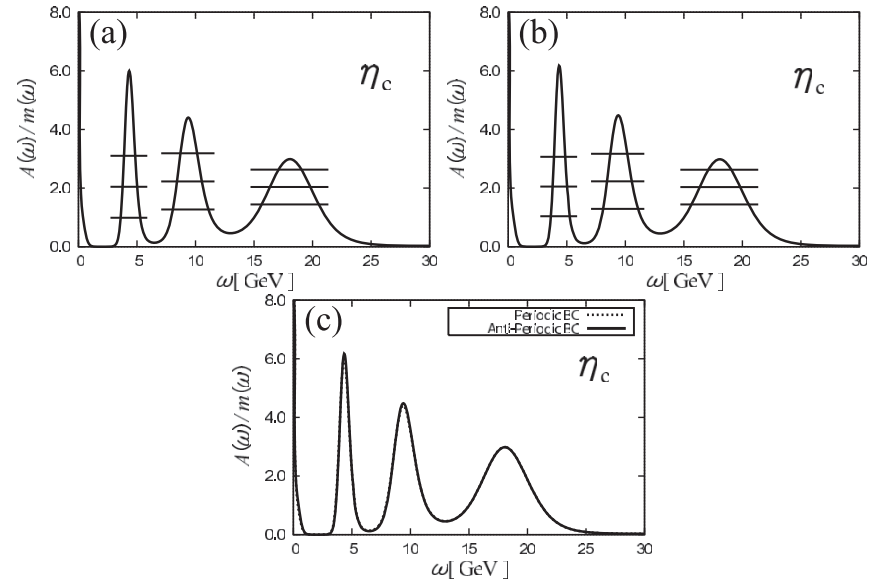

FIG. 10. The spectral function $A(\omega)$ of the $c \bar{c}$ state in pseudoscalar $\left(J^{P}=0^{-}\right)$channel on (a) PBC and (b) APBC at $1.62 T_{c}$ extracted with MEM from lattice QCD data of the temporal correlator. $m(\omega)$ denotes the default function of MEM [23-25]. The statistical error is denoted by the three solid lines. There is a low-lying peak which corresponds to $\eta_{c}\left(m_{\eta_{c}} \simeq 3.0 \mathrm{GeV}\right)$ even above $T_{c}$. We add (c) as the comparison between PBC (dotted line) and APBC (solid line). The results almost completely coincide between PBC and APBC cases. nates from our simple choice of the perturbative default function, since the actual lattice QCD calculation may not reach the perturbative region. In Ref. [23], the authors tune the default function by varying the overall factor to include the nonperturbative renormalization effect phenomenologically. They obtain accurate spectral functions by using a tuned default function: its form is taken from the perturbative default function $m(\omega)=m_{0} \omega^{2}$ for the two-quark system, but the coefficient $m_{0}$ is tuned like an adjustable parameter so as to obtain the best MEM result. They use a larger value of $m_{0}$ than the lowest perturbative one, i.e., $m_{0}=1 / 4 \pi^{2} \times 1.51$ (spin-averaged) for the $J / \Psi$ channel and $m_{0}=3 / 8 \pi^{2} \times 2.89$ for the $\eta_{c}$ channel. In Ref. [25], the authors suggest the difficulty to obtain accurate spectral functions with a default function of a perturbative twoquark spectral function, especially at high temperatures. In their work, they obtain accurate spectral functions by adopting a sophisticated hybrid-type default function above $T_{c}$ : its high-energy part coincides with the spectral function reproduced at $T=0.75 T_{c}$ in lattice QCD, and its low-energy part coincides with the perturbative form of the default function, $m(\omega)=m_{0} \omega^{2}$, where $m_{0}$ is chosen so as to make the default function smoothly connected.

Here, our main aim in this MEM analysis is to compare the spectral functions between PBC and APBC, and, in spite of the statistical error, their coincidence seems to be remarkable, which would indicate the spatially localized nature of $J / \Psi$ and $\eta_{c}$ at $T=1.62 T_{c}$. Also in our MEM analysis, the improvement of the default function is desired to get the solid conclusion, which will be considered in our further study [39].
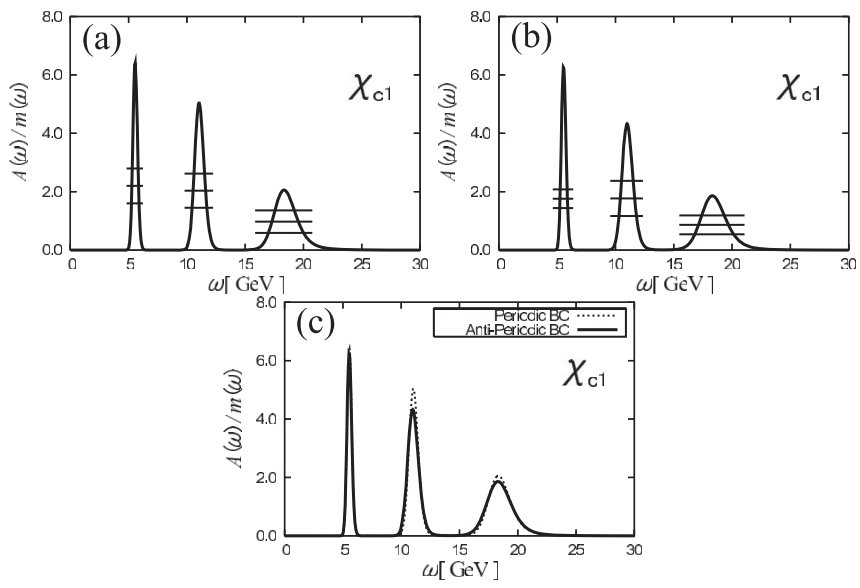

FIG. 11. The spectral function $A(\omega)$ of the $c \bar{c}$ state in axialvector $\left(J^{P}=1^{+}\right)$channel on (a) PBC and (b) APBC at $1.62 T_{c}$ extracted with MEM from lattice QCD data of the temporal correlator. $m(\omega)$ denotes the default function of MEM [23-25]. The statistical error is denoted by the three solid lines. In contrast to the $J / \Psi$ and $\eta_{c}$ cases, there is no low-lying peak which corresponds to $\chi_{c 1}\left(m_{\chi_{c 1}} \simeq 3.5 \mathrm{GeV}\right)$. The peaks in high-energy region $(\omega>5 \mathrm{GeV})$ are considered as lattice artifacts. We add (c) as the comparison between PBC (dotted line) and APBC (solid line). These results almost coincide. 
Next, we investigate the MEM analysis for $\chi_{c 1}$ above $T_{c}$ in lattice QCD. using the local interpolating field of $O_{\chi_{c 1}}=$ $\bar{c} \gamma_{5} \gamma_{k} c$. Figure 11(a) shows the spectral function in $\chi_{c 1}\left(J^{P}=1^{+}\right)$channel on PBC at $1.62 T_{c}$. There is no low-lying peak which corresponds to $\chi_{c 1} \quad\left(m_{\chi_{c 1}} \simeq\right.$ $3.5 \mathrm{GeV}$ ). In fact, in contrast to $J / \Psi$ and $\eta_{c}$, the low-lying structure of the spectral function in $\chi_{c 1}$ channel differs from that in $J / \Psi$ and $\eta_{c}$ channels at $1.62 T_{c}$. Therefore, the MEM analysis indicates that the dissociation of $\chi_{c 1}$ occurs already at $1.62 T_{c}$. In the high-energy region around $6 \mathrm{GeV}$, we can see a sharp peak in the spectral function. Figure 11(b) shows the spectral function in $\chi_{c 1}$ channel on APBC at $1.62 T_{c}$. There is almost no difference between $\mathrm{PBC}$ and APBC. We compare these results in Fig. 11(c) and confirm that the spectral function on $\mathrm{PBC}$ almost coincides with that on APBC. This BC independence indicates that the peak around $6 \mathrm{GeV}$ corresponds to a spatially compact (quasi-)bound state. The state around $6 \mathrm{GeV}$ may be a bound state of doubler(s), as was suggested in Refs. [25,38].

\section{CONCLUSION AND OUTLOOK}

We have investigated $J / \Psi$ and $\eta_{c}$ above $T_{c}$ with anisotropic quenched lattice QCD to clarify whether the $c \bar{c}$ systems above $T_{c}$ are compact (quasi-)bound states or scattering states. We have adopted the standard Wilson gauge action and the $O(a)$-improved Wilson quark action with renormalized anisotropy $a_{s} / a_{t}=4.0$. We have used $\beta=6.10$ on $16^{3} \times(14-26)$ lattices, which correspond to the spatial lattice volume $V \equiv L^{3} \simeq(1.55 \mathrm{fm})^{3}$ and $T=$ (1.11-2.07) $T_{c}$. To clarify whether compact charmonia survive in the deconfinement phase, we have investigated spatial boundary-condition dependence of the energy of $c \bar{c}$ systems above $T_{c}$. In fact, for low-lying $c \bar{c}$ scattering states, it is expected that there appears a significant energy difference $\Delta E \equiv E(\mathrm{APBC})-E(\mathrm{PBC})$ between periodic and antiperiodic boundary conditions as $\Delta E \simeq 2 \sqrt{m_{c}^{2}+3 \pi^{2} / L^{2}}-2 m_{c}$ on the finite-volume lattice. For enhancement of the ground-state overlap, we have used the spatially extended operator with Gaussian function.

As a result, both in $J / \Psi$ and $\eta_{c}$ channels, we have found almost no spatial boundary-condition dependence of the energy of the low-lying $c \bar{c}$ system even on the finitevolume lattice for $(1.11-2.07) T_{c}$. These results indicate that $J / \Psi$ and $\eta_{c}$ survive as spatially compact $c \bar{c}$ (quasibound states for (1.11-2.07) $T_{c}$. Also, the inversion of levels of $J / \Psi$ and $\eta_{c}$ above $1.3 T_{c}$ has been seen. In fact, we have observed the significant reduction of the $J / \Psi$ pole mass of about $100 \mathrm{MeV}$, above $1.3 T_{c}$. Experimentally, it may be interesting to investigate the possible change of the $J / \Psi$ mass above $T_{c}$.

We have also performed the MEM analysis for the $c \bar{c}$ systems in $J / \Psi\left(J^{P}=1^{-}\right), \eta_{c}\left(J^{P}=0^{-}\right)$, and $\chi_{c 1}\left(J^{P}=\right.$ $1^{+}$) channels above $T_{c}$ using the lattice QCD data. For this analysis, we have adopted lattice QCD at $1.62 T_{c}$ with the
Wilson quark action with $\beta=7.0$ and $a_{s} / a_{t}=4.0$. For the $S$-wave channel, we have obtained the same results on the survival of $J / \Psi$ and $\eta_{c}$ as the spatially localized compact bound state above $T_{c}$. In contrast to the $J / \Psi$ and $\eta_{c}$ cases, the spectral function in the $P$-wave channel has no low-lying peak structure corresponding to $\chi_{c 1}$ around $3.5 \mathrm{GeV}$. This fact indicates that $\chi_{c 1}$ already dissociates at $1.62 T_{c}$. The further analysis of charmonia at high temperature with MEM will be reported in Ref. [39].

Through the MEM analysis, we have observed the spatially localized bound state in a high-energy region, which is not affected by the spatial boundary condition at all. The bound state appearing in high-energy region on the lattice would be the bound state of doubler(s), which is unphysical.

Our study of charmonia indicates that the $S$-wave mesons $J / \Psi$ and $\eta_{c}$ survive even above $T_{c}\left(\sim 2 T_{c}\right)$ as compact bound states.

As a successive work, we are performing the same analyses for other charmed mesons, e.g., $D$ mesons. The narrowness of the decay width of charmonia strongly depends on whether the decay channel of $D \bar{D}$ opens or not. If the mass of the $D$ meson is shifted at high temperature, a drastic change of decay width of some charmonia possibly occurs, which may be interesting both theoretically and experimentally [40].

The subject of hadrons in a high density system is fascinating. For example, CERES Collaboration presented interesting events on the lepton pair production in a lowmass region from $p$-Be and $p$ - $\mathrm{Au}(450 \mathrm{GeV} / c)$ and from $S$-Au (200 GeV/c per nucleon) [41]. It is reported that the lepton pairs from $S$-Au in the invariant mass range $0.2 \mathrm{GeV} / c^{2}<m<1.5 \mathrm{GeV} / c^{2}$ are largely enhanced compared with the hadronic contributions, while those from $p$-Be and $p$-Au are not enhanced. The enhancement is considered to be the signal of the change of hadronic nature of vector mesons in finite density system [41-43]. Namely, the mass reduction and/or the width broadenings of vector mesons at finite density can lead to the enhancement of low-energy lepton pairs. At the experimental facility J-PARC (Japan Proton Accelerator Research Complex) in Japan, which is planned to run in 2008, the experiments of a high density system are expected to take place. For these experiments, the theoretical study of a finite density system is required. Then the study at finite density in lattice QCD is challenging and worth trying.

The survival of $J / \Psi$ above $T_{c}$ may change the scenario of $J / \Psi$ suppression. These analyses give us the further knowledge of QCD at finite temperature.

\section{ACKNOWLEDGMENTS}

We thank Professor M. Oka for fruitful discussions and suggestions. H. S. and N.I. are supported in part by the Grant for Scientific Research [(B) No. 15340072 and (C) No. 16540236] from the Ministry of Education, 
Culture, Sports, Science and Technology, Japan. H.I. is supported by the Japan Society for the Promotion of Science for Young Scientists. T.D. is supported by Special Postdoctoral Research Program of RIKEN. K. T. is supported by a 21st Century COE Program at Kyoto University. Our lattice QCD calculations have been performed on NEC-SX5 at Osaka University.
[1] T. Celik, J. Engels, and H. Satz, Phys. Lett. 125B, 411 (1983).

[2] J. B. Kogut, M. Stone, H. W. Wyld, W. R. Gibbs, J. Shigemitsu, S. H. Shenker, and D. K. Sinclair, Phys. Rev. Lett. 50, 393 (1983).

[3] J.I. Kapusta, Finite-Temperature Field Theory (Cambridge University Press, Cambridge, England, 1989), and references therein.

[4] T. Hashimoto, O. Miyamura, K. Hirose, and T. Kanki, Phys. Rev. Lett. 57, 2123 (1986).

[5] T. Matsui and H. Satz, Phys. Lett. B 178, 416 (1986).

[6] T. Hatsuda and T. Kunihiro, Phys. Rep. 247, 221 (1994), and references therein.

[7] H. Ichie, H. Suganuma, and H. Toki, Phys. Rev. D 52, 2944 (1995); S. Sasaki, H. Suganuma, and H. Toki, Phys. Lett. B 387, 145 (1996).

[8] H. Iida, M. Oka, and H. Suganuma, Eur. Phys. J. A 23, 305 (2005).

[9] B. B. Back et al. (PHOBOS Collaboration), Nucl. Phys. A757, 28 (2005).

[10] I. Arsene et al. (BRAHMS Collaboration), Nucl. Phys. A757, 1 (2005).

[11] J. Adams et al. (STAR Collaboration), Nucl. Phys. A757, 102 (2005).

[12] K. Adeox et al. (PHENIX Collaboration), Nucl. Phys. A757, 184 (2005).

[13] M.C. Abreu et al. (NA50 Collaboration), Nucl. Phys. A661, 93 (1999); Phys. Lett. B 477, 28 (2000).

[14] H.J. Rothe, Lattice Gauge Theories (World Scientific, Singapore, 2005), 3rd ed., and references therein.

[15] F. Karsch, J. Phys. G 30, S887 (2004), and references therein.

[16] J. Rafelski and B. Müller, Phys. Rev. Lett. 48, 1066 (1982); 56, 2334(E) (1986)

[17] S. S. Adler et al. (PHENIX Collaboration), Phys. Rev. Lett. 91, 072303 (2003).

[18] X. N. Wang, Phys. Rep. 280, 287 (1997).

[19] C. DeTar and J. B. Kogut, Phys. Rev. Lett. 59, 399 (1987); Phys. Rev. D 36, 2828 (1987).

[20] A. Nakamura and S. Sakai, Phys. Rev. Lett. 94, 072305 (2005).

[21] T. Hirano and Y. Nara, Phys. Rev. Lett. 91, 082301 (2003).

[22] T. Umeda, K. Katayama, O. Miyamura, and H. Matsufuru, Int. J. Mod. Phys. A 16, 2215 (2001); H. Matsufuru, O. Miyamura, H. Suganuma, and T. Umeda, AIP Conf. Proc. 594, 258 (2001).

[23] M. Asakawa and T. Hatsuda, Phys. Rev. Lett. 92, 012001 (2004).

[24] T. Umeda, K. Nomura, and H. Matsufuru, Eur. Phys. J. C 39, 9 (2005)
[25] S. Datta, F. Karsch, P. Petreczky, and I. Wetzorke, Phys. Rev. D 69, 094507 (2004); J. Phys. G 31, S351 (2005).

[26] H. Iida, T. Doi, N. Ishii, and H. Suganuma, Proc. Sci., LAT2005 (2005) 184 [hep-lat/0509129].

[27] N. Ishii, T. Doi, H. Iida, M. Oka, F. Okiharu, and H. Suganuma, Phys. Rev. D 71, 034001 (2005); N. Ishii, T. Doi, Y. Nemoto, M. Oka, and H. Suganuma, Phys. Rev. D 72, 074503 (2005).

[28] M. Lüscher, Nucl. Phys. B354, 531 (1991).

[29] N. Mathur, F.X. Lee, A. Alexandru, C. Bennhold, Y. Chen, S. J. Dong, T. Draper, I. Horvath, K. F. Liu, S. Tamhankar, and J.B. Zhang, Phys. Rev. D 70, 074508 (2004).

[30] T. T. Takahashi, T. Umeda, T. Onogi, and T. Kunihiro, Phys. Rev. D 71, 114509 (2005).

[31] M. Gao, Phys. Rev. D 41, 626 (1990).

[32] T. T. Takahashi, H. Matsufuru, Y. Nemoto, and H. Suganuma, Phys. Rev. Lett. 86, 18 (2001); Phys. Rev. D 65, 114509 (2002); H. Matsufuru, Y. Nemoto, T. T. Takahashi, T. Umeda, Quantum Chromodynamics and Color-Confinement, edited by $\mathrm{H}$. Suganuma, M. Fukushima, and H. Toki (World Scientific, Singapore, 2000), p. 103.

[33] K. Yagi, T. Hatsuda, and Y. Miake, Quark-Gluon Plasma (Cambridge University Press, Cambridge, England, 2005).

[34] T. R. Klassen, Nucl. Phys. B533, 557 (1998); H. Matsufuru, T. Onogi, and T. Umeda, Phys. Rev. D 64, 114503 (2001).

[35] N. Ishii, H. Suganuma, and H. Matsufuru, Phys. Rev. D 66, 094506 (2002); 66, 014507 (2002).

[36] A.X. El-Khadra, A.S. Kronfeld, and P. B. Mackenzie, Phys. Rev. D 55, 3933 (1997).

[37] M. Asakawa, Y. Nakahara, and T. Hatsuda, Prog. Part. Nucl. Phys. 46, 459 (2001).

[38] T. Yamazaki, S. Aoki, R. Burkhalter, M. Fukugita, S. Hashimoto, N. Ishizuka, Y. Iwasaki, K. Kanaya, T. Kaneko, Y. Kuramashi, M. Okawa, Y. Taniguchi, A. Ukawa, and T. Yoshie (CP-PACS Collaboration), Phys. Rev. D 65, 014501 (2001).

[39] K. Tsumura et al. (unpublished).

[40] A. Hayashigaki, Phys. Lett. B 487, 96 (2000).

[41] G. Agakichiev et al. (CERES Collaboration), Phys. Rev. Lett. 75, 1272 (1995).

[42] V. L. Eletsky, B. L. Ioffe, and J. I. Kapusta, Eur. Phys. J. A 3, 381 (1998); V. L. Eletsky and B. L. Ioffe, Phys. Rev. Lett. 78, 1010 (1997); Phys. Lett. B 477, 28 (2000).

[43] H. Iida, M. Oka, and H. Suganuma, Nucl. Phys. B, Proc. Suppl. 141, 191 (2005). 